\title{
Herausgegeben von Bernd Dörflinger, Claudio La Rocca, Robert Louden y Ubirajara Rancan de Azevedo Marques (eds.) (2015). Kant's Lectures/Kants Vorlesungen. Berlín-Boston: Walter de Gruyter.
}

El libro Kant's Lectures/Kants Vorlesungen reúne un conjunto de trabajos que fueron elaborados a partir de la presentación y discusión de los mismos en el VIII Kant Kolloquium que tuvo lugar en Marilia, Brasil, entre el 18 y el 21 de agosto de 2013.

Tal como lo indica el título, su objeto de estudio está constituido por las Lecciones o clases dictadas por Kant. Ellas dan testimonio de la actividad pública del filósofo y son una fuente fundamental para el estudio de la evolución de su pensamiento, ya que profundizan y complementan aspectos de su doctrina, tal como la conocemos a través de sus obras publicadas. Los trabajos se orientan según distintas líneas de investigación: histórica y especulativo-conceptual. El texto brinda abundante material para el estudio del pensamiento de Kant, tanto respecto a las fuentes, como a la discusión con los filósofos de la época $\mathrm{y}$, también, con respecto a la elaboración de su propio pensamiento, que él mismo ha ido efectuando en la medida en que exponía los temas y los autores. Algunos trabajos están escritos en inglés y otros en alemán.

El libro nuclea a destacados investigadores de América y Europa y comienza exponiendo los elementos históricos fundamentales para ubicar las Lecciones en el conjunto del pensamiento de Kant. A partir de este marco general, sumamente preciso y riguroso, se presentan los capítulos dedicados a las distintas Lecciones. El primero de ellos está centrado en las Lecciones de Lógica, el capítulo dos está dedicado a las Lecciones de Antropología, el capítulo tres aborda las Lecciones de Ética, el capítulo cuatro se centra en las Lecciones de Derecho, el capítulo cinco está dedicado a las Lecciones de Metafísica y el último capítulo trata sobre las Lecciones de Teología racional.

El primer artículo que se presenta en el libro es el de Werner Stark: Ensayo de un informe sumario y detallado sobre las Lecciones de Immanuel Kant. Allí el autor plantea una reconstrucción del marco histórico en el que se desenvuelven las Lecciones de Kant, en la segunda mitad del siglo XVIII. El primer punto traza la estructura de la universidad y sus transformaciones en ese período. Presenta los rasgos principales de la fundación de la universidad Albertina, para ubicar allí la praxis docente de Kant. En el segundo punto presenta Lecciones de Kant y enuncia cuáles son los manuales que éste ha utilizado como base de las exposiciones. 
Destaca los períodos y la secuencia en que Kant las ha dictado, la elección de los temas y sugiere criterios subyacentes a esa decisión. Señala las cuestiones de datación inherentes a la Edición Académica y realiza un amplio relevamiento de los problemas que se derivan de allí. El artículo constituye una investigación histórica precisa, y erudita del marco en el que se desenvuelven las Lecciones de Kant.

En el primer capítulo dedicado a las Lecciones de Lógica, la investigación de María Jesús Lobeiras, bajo la pregunta: ¿Por qué son interesantes las Lecciones de Lógica de Kant? considera, en su primera parte, la importancia que tienen las Lecciones para un estudio de la historia del desarrollo y de las fuentes del pensamiento de Kant. Presenta la clasificación de los escritos de Kant en el interior de la "Akademie Ausgabe", aborda los problemas filológicos y el valor de las Lecciones. En la segunda parte, se aborda la cuestión de los motivos por los que son interesantes las Lecciones de Lógica. Por medio de ejemplos cuidadosamente elegidos muestra que ellas pueden suministrar claves para la comprensión del pensamiento de Kant. A la vez, dado que el Compendio de Lógica de Meier, elegido por Kant para sus clases, representa la cultura de la Ilustración, se puede saber cómo ha sido la apropiación que ha hecho Kant de ésta. Así, las Lecciones de lógica no sólo son interesantes para los intérpretes del pensamiento de Kant, sino, también, para quienes se ocupan de la Ilustración alemana.

Catalina Gonzales en su artículo: Kant, Cicerón y "popularidad" en las Lecciones de Lógica sostiene que Kant expresa una valoración común en la modernidad acerca del status inferior de la oratoria en relación a la poesía. Al definir a la oratoria como arte dialéctica la despoja del juego de las facultades, que es la base del juicio estético. Pero, Kant también se refiere a la oratoria como el arte de hablar bien o elocuencia y, en esto, reside su valor positivo. Este aspecto está vinculado a Cicerón y a Quintillano, al arte del vir bonus. La autora recurre a las Lecciones de Lógica donde, a su criterio, podemos encontrar mayor elaboración del arte de la elocuencia, basada en la noción de popularidad. Con los resultados extraídos de las Lecciones de Lógica retoma el tema de la estimación o desestimación de la retórica como arte bello desde el análisis del ideal de oratoria del vir bonus dicendi peritus y su vínculo con la Ilustración.

Silvia Altmann en su artículo: Notas acerca de la materia y el contenido de los conceptos, después de una introducción en la lógica transcendental en donde muestra la necesaria referencia de los conceptos puros a la sensibilidad, facultad que provee la materia para esos conceptos, 
se pregunta ¿qué debemos entender por contenido y materia de un concepto? Propone recurrir a la Lógica Jäsche para pensar los conceptos de forma, materia y contenido. El propósito del artículo es mostrar que, en las Lecciones de Lógica, Kant distingue suficientemente el contenido de un concepto de la materia de un concepto, puesto que contenido no es materia sino aquello que en el concepto nos permite representar al objeto, que es su materia. Recorre en su argumentación pasajes de la Lógica Viena y de la Lógica Blomberg.

Joao Carlos Brum Torres, en su artículo: Notas sobre el concepto kantiano de "concepto empírico" parte de la contraposición entre una teoría clásica del concepto como representación y una nueva teoría de la posesión de conceptos. El propósito del artículo consiste en mostrar cómo puede ser ubicado el análisis del concepto de concepto empírico de Kant con respecto a los problemas de la cognición conceptual, que son puestos de relieve en las nuevas teorías. A su vez, también, evaluar si corresponde ubicar el análisis que realiza Kant, en la posición clásica. En la segunda parte del artículo, el autor analiza la función de las categorías y de los conceptos de reflexión en la teoría kantiana de la formación de los conceptos, en el contexto de la Crítica de la razón pura con referencias a la Lógica Jäsche, a la Lógica Viena y a la Lógica Wundlacken.

Andrea Faggion en su trabajo plantea la pregunta: ¿Pueden las solas intuiciones representar objetos? Y la plantea como equivalente a la formulación contemporánea ¿existen contenidos no conceptuales? La autora defiende en el artículo una teoría kantiana del conocimiento conceptualista de caracteres propios y peculiares, que acepta un enunciado no conceptualista, pero no contenidos no conceptuales. Mostrará, recurriendo a la Logica Jäsche y a la Deducción Trascendental A, que este enunciado no conceptualista es compatible con el "refinado conceptualismo de la Deducción transcendental".

En el capítulo II se aborda el estudio de las Lecciones de Antropología. Leonel Ribeiro Dos Santos, en su artículo: La economía y la teleología del mal en las Lecciones de Antropología de Kant considera cuatro transcripciones de las mismas: Antropología Friedländer, Pillau, Menschenkunde y Mrongovius. En ellas se afirma que la cuestión del mal va unida a la condición humana y, en este punto, se plantea la coincidencia de Kant con Rousseau. Sin embargo, esa coincidencia es un punto de partida para superar, lo que Kant llama, las "paradojas" de Rousseau. El autor investiga en el artículo la función que cumple la maldad humana "en la economía de la naturaleza en relación a la especie humana y al destino 
del hombre como especie". Para ello, vincula las distintas versiones de las Lecciones de Antropología con otros escritos de Kant, en los que el filósofo asume el principio teleológico.

Fernando Silva en su artículo: "Para la invención se requiere ingenio". Acerca de la evolución del concepto de ingenio en las Lecciones de Antropología de Kant se propone investigar la elaboración que hace Kant del concepto de ingenio, a través de las distintas versiones de las Lecciones y evaluar el aporte de Kant a la evolución de este concepto. Considera la oposición que estable Kant entre ingenio y facultad de Juzgar (Urteilskraft) y muestra la diferencia entre las actividades de la imaginación y del entendimiento, que se corresponden con el accionar diferente del ingenio y de la facultad de juzgar. El autor aborda, en la segunda parte del artículo, la relación entre ingenio y facultad de juzgar con la poesía y la filosofía respectivamente. Presenta una conexión profunda entre ingenio, poesía y filosofía, a partir de las indicaciones de Kant en la Lección del semestre de invierno 1772-3.

Joãosinho Beckenkam en su artículo: Kant y Gerard acerca de la imaginación plantea en qué medida la concepción de imaginación productiva de Kant se inspira en la idea de imaginación activa de Gerard. Analiza la concepción de imaginación activa de Gerard y, bajo esa concepción, interpreta la idea de genio. El texto se focaliza en la idea de "imaginación activa" como precursora de la idea de "imaginación productiva" de Kant. Toda la exposición, documentada con citas textuales de ambos autores, conduce a mostrar que la referencia de Kant a Gerard en la Antropología Menschenkunde con respecto a la "imaginación productiva", no alude a una relación meramente externa, sino que la expresión "imaginación activa" pone de relieve muchas determinaciones que Kant concibe bajo la denominación de "imaginación productiva".

Heiner Klemme en su artículo: Conocimiento, sentimiento, deseo -posesión de sí mismo. Reflexiones sobre la conexión de las facultades en la doctrina del imperativo categórico de Kant plantea que, para comprender, desde la ética kantiana, cómo y por qué juzgamos como morales a las personas y a las acciones es necesario no sólo entender la fundamentación de la ley moral en la razón práctica sino también la condición sensible del ser humano, sus deseos, sus sentimientos y la relación de estos con la ley moral. El autor analiza la conexión que existe entre nuestros sentimientos y afecciones, por un lado, y la razón pura práctica, por el otro. El análisis se basa en particular en la Antropología Mrongovius, en la Fundamentación para una Metafísica de las costumbres y en la Crítica de la 
razón práctica. Un ítem fundamental de este análisis es la diferencia entre el sentimiento moral, como el efecto de la razón pura sobre el ánimo, y el sentimiento meramente sensible. En esta constelación se analiza el concepto de self-possession.

Nuria Sánchez Madrid en su artículo: Prudencia y reglas para guiar la vida. El desarrollo de la normatividad pragmática en las Lecciones de Antropología de Kant aborda, en primer lugar, la relación del conocimiento antropológico con la doctrina moral de Kant. En segundo lugar, propone la reconstrucción de la unidad del material disperso, para pensar la orientación de la acción de un hombre de mundo. La tesis de la autora es que "las Lecciones de Antropología de Kant muestran una evolución que conduce a un incremento de la conciencia acerca del significado y los límites de la normatividad pragmática". Analiza textos de la Antropología Feyerabend, la Antropología Collins, Menschenkunde y Busolt. Culmina confrontando el lugar que ocupan las Lecciones de Antropología en relación al conjunto del sistema de la filosofía de Kant y fundamenta por qué la normatividad pragmática está siempre sujeta a principios morales.

En el capítulo III dedicado a las Lecciones de Ética se encuentra el artículo de Robert Louden: Moralidad para los seres humanos: la teoría ética en la Lección Vigilantius de Kant. En el primer punto se presenta la figura de Johann Friedrich Vigilantius (1757-1823) y se plantea la cuestión acerca de cómo pensar el vínculo entre la Lección Vigilantius y la obra posteriormente publicada con el título Metafísica de las costumbres (1797). En un segundo punto se comparan ambos escritos. En la Lección Vigilantius Kant trabaja con una concepción de la teoría ética focalizada en los seres humanos en particular, en lugar del ser racional como tal. El autor señala a la Lección Vigilantius como un giro en la concepción de Kant acerca de lo que es una metafísica de la moral. El último punto aborda una discusión, presente al final de la Lección Vigilantius y al final de la Metafísica de las Costumbres, acerca de la religión y los deberes frente a Dios.

El capítulo IV dedicado a las Lecciones de Derecho presenta el trabajo de Günter Zöller: "Sin esperanza ni miedo" El derecho natural Feyerabend de Kant, sobre el fundamento de la obligatoriedad de una acción presenta la cuestión de la transferencia del concepto de obligatoriedad desde la esfera del derecho y la política a la esfera de la moral y la ética. El autor examina, en Kant, la transferencia de conceptos fundamentales del derecho que se adoptan metodológicamente en la ética. Como 
central analiza el concepto de obligatoriedad. Kant elabora el paralelo sistemático entre derecho y ética en el curso de la formación de su filosofía práctica. Testimonio de ello son las Lecciones sobre el derecho natural Feyerabend, que coinciden temporal y temáticamente con las investigaciones de Kant en la Fundamentación para una metafísica de las costumbres. El autor compara ambos textos y realiza un examen detallado de esa diferenciación mostrando el aporte específico que se extrae de las Lecciones sobre el derecho natural Feyerabend. Finaliza señalando la analogía estructural entre derecho y ética que se extrae de estas Lecciones.

El capítulo V dedicado a las Lecciones de Metafísica de Kant comienza con el trabajo de Jacinto Rivera de Rosales titulado Kant: el mundo teórico de la metafísica $L_{1}$ (1776-1778). El autor muestra el estado de desarrollo del pensamiento de Kant en la década silenciosa mediante el testimonio que representan esas Lecciones de Metafísica y lo presenta en relación al desarrollo que tendrán en la Crítica de la razón pura. En la primera parte ubica temporalmente a las Lecciones que se conocen como Metafísica $L_{1}$. En el segundo punto aborda la Estética transcendental y atiende al estado de la investigación de Kant en ese período, con respecto a sus conceptos fundamentales. En el tercer punto, considera a los conceptos de la Analítica Transcendental y señala las diferencias de su desarrollo en ambos momentos. En un cuarto punto, examina los conceptos de la Dialéctica, luego, considera la idea y división de la metafísica y finaliza con una exploración del concepto de mundo inteligible como una hipótesis necesaria para la razón en sus dos usos.

El artículo de Gualtiero Lorini: La contribución de las Lecciones de Metafísica de Kant para una mejor comprensión de la arquitectónica parte de señalar la relación oscilante que existe entre los términos ontología y filosofía transcendental y muestra que las Lecciones proveen elementos que hacen inviable la simple sustitución de ontología por filosofía transcendental. En el segundo punto, aborda Las Lecciones sobre la Enciclopedia filosófica, en el que es evidente la exigencia sistemática. Luego, analiza la relación entre crítica y filosofía transcendental considerando, en primer lugar, el texto de la Metafísica Mrongovius (1783) y, luego, los textos de la Metafísica Pölitz $\mathrm{L}_{2}(1790-1791)$ y de la Metafísica Vigilantius (1794-1795). Muestra que, de acuerdo con la necesidad arquitectónica de la Crítica de la razón pura y, a diferencia de lo que planteaba Kant en la Enciclopedia, crítica y filosofía transcendental están separadas. Finalmente, aborda el rol que juegan las Lecciones en el marco de la reforma kantiana a la metafísica. 
El trabajo de Juan Bonaccini: La consideración de Kant de los milagros en sus Lecciones de Metafísica, señala que Kant aborda el problema de los milagros a partir de la interpretación de la cosmología de Baumgarten. Para evaluar en qué medida Kant está preocupado por los milagros en sus Lecciones de Metafísica, el autor señala dos ítems: uno es la lectura y el comentario que Kant hace de Baumgarten y, el otro: la lectura sobre Baumgarten acerca de los milagros en las Lecciones: Metafisica $L_{1}$ (Pölitz), Metafísica Mrongovius, Metafísica Dohna y Metafísica $K_{2}$. El problema gira en torno a determinar si Kant ofrece una teoría propia acerca de los milagros. La tesis del autor es que Kant sostiene su propia teoría, cuyo punto central reside en explicar en qué sentido ellos pueden ser posibles sin contradecir el orden de la naturaleza.

Ubirajara Rancán de Azevedo Marques en su artículo: Sobre la epigénesis: observaciones históricas y filológicas, emprende una exposición histórica, filológica y filosófica del concepto de epigénesis. La investigación se presenta en forma de Observaciones. En la primera de ellas, se ubica el término históricamente en el marco de la ciencia de la naturaleza. En la Observación dos, se hace un relevamiento del término en el conjunto de la obra de Kant y se enuncian algunos problemas de interpretación. En la observación tres el autor se ocupa del término epigénesis en el contexto de la deducción transcendental B. En la Observación cuarta, analiza el término preformación genérica y examina el concepto de epigénesis como teoría de la preformación general. Desde aquí interpreta el $\S 27$ de la Crítica de la razón pura. El artículo sitúa el problema de la epigénesis en la obra de Kant y en relación a la discusión de su época.

El último capítulo del libro está dedicado a las Lecciones de Teología racional y en él se presenta el artículo de Bern Dörflinger: Sobre el estado de desarrollo de la Teología racional de Kant en su Lección del semestre de invierno de 1783/84. El mismo articula la investigación histórica y el análisis sistemático, sobre la base de la pregunta acerca del grado de elaboración de la doctrina de la ético-teología, de la doctrina del bien supremo en el marco de esa lección. Para su análisis, el autor define un punto de partida y un punto de llegada, decisión que justifica a lo largo de la exposición, y marca los hitos que considerará: la exposición de los pasajes relevantes de la Crítica de la razón pura, incluida la Doctrina del método; la doctrina de los postulados de la Crítica de la razón práctica; las partes relevantes de la Doctrina de Método de la Crítica de la facultad de juzgar y el posicionamiento final en los Escritos de Religión. Así define 
un arco que va desde la posición presentada por Kant en la primera Crítica y, una posición posterior, en la que las leyes morales aún sin la aceptación de un Dios siguen siendo válidas sin límite.

Todos los artículos de este volumen están muy bien escritos, lógicamente muy bien organizados y muestran en forma rigurosa aspectos de la filosofía de Kant a los que se puede acceder mediante las Lecciones. Ellas permiten profundizar en conceptos fundamentales de la filosofía kantiana e incluso problematizar temas que aparecen en distintas partes de la obra de Kant o descubrir conexiones que pasan inadvertidas, cuando sólo se consideran las obras publicadas. Hay un hilo lógico que recorre el volumen, esto indica que ha sido muy bien pensada la estructura general de la obra y la temática seleccionada y abordada por los distintos autores. Se muestra una concatenación de los textos, de modo que algunos problemas que son mencionados en un artículo, son desarrollados en otro. Todos ellos cubren un amplio panorama de los temas y problemas desarrollados por Kant en sus clases, así como también, dan cuenta de su vinculación con la obra publicada del filósofo. Las "Vorlesungen" ofrecen material para la elaboración sistemática de problemas cruciales de la filosofía de Kant. Al respecto, este libro constituye un estudio histórico y sistemático-conceptual de las "Lectures", realizado con rigurosidad, bien documentado y con una exégesis erudita de los textos abordados, que lo convierte en un material necesario para ampliar y profundizar el estudio del pensamiento de Kant y de conceptos fundamentales de la filosofía moderna.

Silvia del Luján Di Sanza

Escuela de Humanidades Universidad Nacional de San Martín sdisanza@hotmail.com 